\title{
BER Analysis of BPSK-SIM based SISO and MIMO FSO systems in strong turbulence with pointing errors
}

\author{
K. Prabu (Corresponding Author) \\ Department of Electronics and Communication Engineering, \\ National Institute of Technology (NIT) \\ Tiruchirappalli-620015, India. \\ E-mail: nitprabu@gmail.com \\ Tel: (91) 9884888408
}

\section{Sriram Kumar}

Department of Electronics and Communication Engineering, National Institute of Technology (NIT)

Tiruchirappalli-620015, India.

\author{
Reza Malekian \\ Department of Electrical, Electronic \& Computer Engineering, \\ University of Pretoria, South Africa.
}

\begin{abstract}
Free Space Optics (FSO) is one of the sprouting technologies in optical communication systems domain. It can be employed as an alternative for the conventional radio frequency $(\mathrm{RF})$ links to work out the current limitations in communication systems. But, the major drawback in FSO communication is the effect of random environment conditions on its performance. In this paper, we analyze the bit error rate (BER) and outage performance of single-input single-output (SISO) and multiple-input multiple-output (MIMO) FSO systems in strong atmospheric turbulence using binary phase shift keying subcarrier intensity modulation (BPSK-SIM) signaling technique. The closed form expressions are derived and the results are realized in terms of 2-D and 3-D plots.

Key words - Free Space Optics, bit error rate, outage probability, single-input single-output, multiple-input multiple-output.
\end{abstract}




\section{Introduction}

Free Space Optics (FSO), also known as open-air photonics or optical wireless or infrared broadband, is a full-duplex line-of-sight (LOS) technology. The technology is extremely useful where landline connection is not feasible. FSO communication employs modulated low-powered visible or infrared (IR) lasers for transmitting data from point-to-point or multipoint through the atmospheric channel. Customarily, laser beams are employed, however non-lasing sources like light-emitting diodes (LEDs) or IR-emitting diodes (IREDs) can also be employed instead [1].

Each FSO wireless system uses an optical source, a lens or telescope that transmits light through the atmospheric channel to another lens receiving the data. FSO systems can operate over expanses of several kilometers even if there is no direct LOS, by employing tactically positioned mirrors to reflect the energy. As the fact, light is transmitted through air faster than glass, so FSO technology offers optical communication at the speed of light. Also, the photons transmitted by the laser are much quicker than electrons moving in a wire and they can pass straight through one another, whereas charge-bearing electrons cannot. As a result, large volumes of data can be transmitted through a confined space in FSO which is not possible to realize in other communication techniques [2].

Unlike conventional RF links, FSO communication is cost-effective, easily upgradable, and immune to radio frequency interference or saturation, serves higher bandwidth at a faster speed and requires no RF spectrum licensing or security software upgrades [3]. And, hence this technology is sprouting as an appealing alternative to the present communication systems. So, FSO can be employed for applications in building-to-building connectivity, disaster recovery, network redundancy and temporary connectivity for applications such as voice and data, video services, medical imaging, fixed-line carrier bypass, CAD and engineering services $[4,5]$.

An FSO communication system is a powerful technique to deploy wireless networks with high transmission rates like wired optical system. But, the communication using FSO depends upon the concern 
of the nature, most significant components being rain, dust, snow, fog, or smog. Due to these random environmental conditions, the transmitted photons in the signals get faded, absorbed, scattered, diffracted and also sometimes gets lost in the channel [6]. This can block the transmission path or even shut down the network, thus impairing the signal strength and performance of the system. MIMO system is useful for resisting temporary link blockage by birds and misalignment when merged with a wide laser beam width and hence eliminates the need for an active tracking. Various diversity techniques are also implemented to improve the link performance at relatively cheaper costs. It is employed by creating several independent paths between the transmitter and receiver. Since, each path fades independently, there is a low chance of fading together. The performance of spatial MIMO systems was studied in [7-10]. Here, we employ the hybrid binary phase shift keying based subcarrier intensity modulation (BPSK-SIM) technique.

The spatial correlation between a pair of constituent transmitters in an MIMO system is investigated in [11]. In a recent work, a coherent MIMO architecture was proposed for FSO communication and BER expressions are derived using a series expansion for gamma-gamma, K-distributed, and negative exponential turbulences [12]. The performance of BPSK-SIM based SISO system is analyzed and its parameters such as average BER, outage probability and channel capacity were evaluated under the strong atmospheric turbulence channel with pointing errors [13]. In this paper, we examine bit error rate (BER) performance of the FSO system with two transmission topologies, single-input-single-output (SISO) and multiple-input-multiple-output (MIMO) in strong atmospheric turbulence with pointing errors.

The paper is organized as follows: Section 2 discusses the SISO and MIMO system models. In Section 3, the gamma-gamma channel model with pointing errors for SISO and MIMO systems are discussed. In Section 4, expressions for average BER of the SISO and MIMO systems are derived and presented. Section 5 describes the numerical results with graphical analysis. Section 6 concludes the paper by summarizing the important results. 


\section{System models}

\subsection{Single-input-single-output system model}

The FSO system considered here, has one transmitting and one receiving apertures. The signals to be transmitted are modulated by using BPSK-SIM signaling technique and transmitted via telescope into discrete time ergodic channel with additive white Gaussian noise (AWGN). The transmitted signal gets scattered due to natural turbulence in the atmospheric channel. These corruptions of signals are triggered by various environmental elements. In this work, we consider strong atmospheric turbulence channel. The signal $(y)$ received at the receiver [14] can be expressed as

$$
y=h \gamma P_{F S O} x+n
$$

where $h$ is the channel state, $\gamma$ is the detector responsivity, $x$ is the transmitted signal, $n$ is the noise caused by various sources and $P_{F S O}$ is the average optical transmitted power. The channel state ' $h$ ' models the optical intensity fluctuations resulting from atmospheric loss, turbulence and fading as [15],

$$
h=h_{l} h_{s} h_{p}
$$

where $h_{l}$ is the attenuation due to beam extinction and path loss, $h_{s}$ due to scintillation effects and $h_{p}$ due to the geometric spread and pointing errors.

Also the signal to noise ratio (SNR) of the received signal considered in [16] is expressed as

$$
\operatorname{SNR}(h)=\frac{(\gamma h)^{2}}{2 \sigma^{2}}
$$

where $\sigma_{n}^{2}$ is the variance of the channel noise, $A$ is the amplitude of the subcarrier signal.

\subsection{Multiple-input-multiple-output system model}

The FSO system considered here, has $M$ transmitting and $N$ receiving apertures. The transmitted signals are BPSK-SIM modulated and transmitted via telescope into discrete time ergodic channel with additive white Gaussian noise (AWGN) in strong atmospheric turbulence. The signal $\left(y_{n}\right)$ received at the receiver can be expressed as [17],

$$
y_{n}=x \gamma \sum_{m=1}^{M} h_{m n}+v_{n}, n=1,2, \ldots . N
$$


where $x$ is the transmitted signal, $\gamma$ is the detector responsivity, $h_{m n}$ is the irradiance from the $m^{\text {th }}$ transmitter and $n^{\text {th }}$ receiver, $v_{n}$ is the AWGN with zero mean and variance $\sigma_{v}=N_{0} / 2$. The irradiance ' $h_{m n}{ }^{\prime}$ models the optical intensity fluctuations resulting from atmospheric loss, turbulence and fading [15] is given by

$$
h_{m n}=h_{l_{m n}} h_{s_{m n}} h_{p_{m n}}
$$

where $h_{l_{m n}}$ is the attenuation due to beam extinction and path loss from the $m^{\text {th }}$ transmitter and $n^{\text {th }}$ receiver, $h_{s_{m n}}$ due to scintillation effects and $h_{p_{m n}}$ due to the geometric spread and pointing errors.

\section{Channel models}

\subsection{Single-input-single-output channel model}

The strong atmospheric turbulence channel is demonstrated employing the combined effects of atmospheric turbulence and pointing errors. The probability density function (PDF) of the considered channel model for SISO system [13] is expressed using the Meijer G function as

$$
f_{h}(h)=\frac{\alpha \beta \xi^{2}}{A_{0} h_{l} \Gamma(\alpha) \Gamma(\beta)} G_{1,3}^{3,0}\left[\left.\frac{\alpha \beta h}{A_{0} h_{l}}\right|_{-1+\xi^{2}, \alpha-1, \beta-1} \xi^{2}\right]
$$

where $\alpha$ and $\beta$ represent the effective number of large and small scale turbulent eddies, $\Gamma(\cdot)$ is the gamma function.

\subsection{Multiple-input-multiple-output channel model}

The probability density function (PDF) of the considered channel model for MIMO systems is expressed using the Meijer $\mathrm{G}$ function as

$$
f_{h_{m n}}\left(h_{m n}\right)=\frac{\alpha_{m n} \beta_{m n} \xi_{m n}^{2}}{A_{0_{m n}} h_{l_{m n}} \Gamma\left(\alpha_{m n}\right) \Gamma\left(\beta_{m n}\right)} \times G_{1,3}^{3,0}\left[\left.\frac{\alpha_{m n} \beta_{m n} h_{m n}}{A_{0_{m n}} h_{l_{m n}}}\right|_{-1+\xi_{m n}^{2}, \alpha_{m n}-1, \beta_{m n}-1} ^{\xi_{m n}^{2}}\right]
$$

where $\alpha_{m n}$ and $\beta_{m n}$ represent the effective number of large and small scale turbulent eddies, $\Gamma(\cdot)$ is the gamma function. 


\section{Average BER}

\subsection{Single-input-single-output system}

For a BPSK-SIM signaled communication system, the conditional BER probability depends on the fluctuation intensity $[18,19]$ and can be expressed as

$$
P_{e c, S I S O}(h)=0.5 \times \operatorname{erfc}\left(\frac{h \gamma}{2 \sigma}\right)
$$

where $\gamma$ is the photo detector responsivity, $\sigma^{2}$ is the variance of the channel noise.

For a gamma-gamma channel with pointing errors, the average BER $P_{e, \text { SISO }}$ can be realized by averaging Eqn. (8) over the PDF of $h$ can be estimated as

$$
P_{e, S I S O}=\int_{0}^{\infty} P_{e c, S I S O}(h) f_{h}(h) d h
$$

By using Eqn. (6) and (8) in (9), the average BER can be obtained as

$$
P_{e, S I S O}=\frac{\alpha \beta \xi^{2}}{A_{0} h_{l} \Gamma(\alpha) \Gamma(\beta)} \times \int_{0}^{\infty} 0.5 \times \operatorname{erfc}\left(\frac{h \gamma}{2 \sigma}\right) \times G_{1,3}^{3,0}\left[\left.\frac{\alpha \beta h}{A_{0} h_{l}}\right|_{-1+\xi^{2}, \alpha-1, \beta-1} \xi^{2}\right] d h
$$

By expressing the $\operatorname{erfc}(\cdot)$ as Meijer G function [20, Eq. (8.4.14.2)], the average BER of BPSK-SIM can be expressed in a closed-form utilizing [21, Eq. (21)].

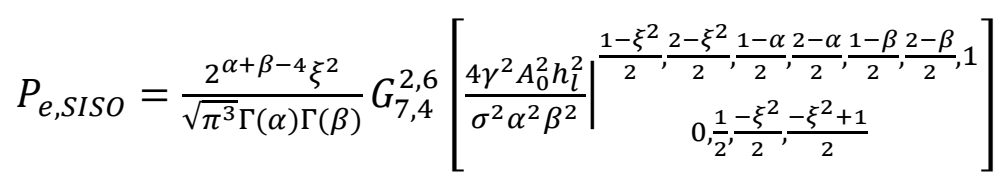

The above average BER expression is expressed in terms of average SNR as

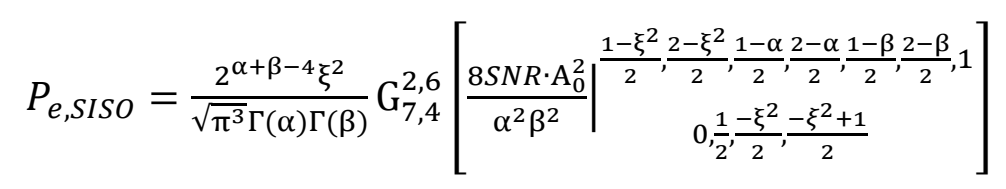

\subsection{Multiple-input-multiple-output system}

The primary solution for a strong atmospheric turbulence channel is spatial diversity technique, which can be applied either at the transmitter (MISO) or at the receiver (SIMO) or at both the sides (MIMO). The average BER for MIMO-FSO links can be calculated from [17] 


$$
P_{e, M I M O}=\int_{0}^{\infty} f_{\boldsymbol{h}}(\boldsymbol{h}) \times 0.5 \times \operatorname{erfc}\left(\frac{h \gamma}{2 M N \sigma} \sqrt{\sum_{n=1}^{N}\left(\sum_{m=1}^{M} h_{m n}\right)^{2}}\right) d \boldsymbol{h}
$$

where $f_{\boldsymbol{h}}(\boldsymbol{h})$ is the joint PDF of vector $\boldsymbol{h}=\left(h_{11}, h_{12}, \ldots h_{M N}\right)$ of length MN.

By substituting Eqn. (7) in (13), we get

$$
\begin{aligned}
P_{e, M I M O}= & \frac{\alpha_{m n} \beta_{m n} \xi_{m n}^{2}}{A_{0_{m n}} h_{l_{m n}} \Gamma\left(\alpha_{m n}\right) \Gamma\left(\beta_{m n}\right)} \times \\
& \int_{0}^{\infty} 0.5 \operatorname{erfc}\left(\frac{h \gamma}{2 M N \sigma} \sqrt{\sum_{n=1}^{N}\left(\sum_{m=1}^{M} h_{m n}\right)^{2}}\right) G_{1,3}^{3,0}\left[\left.\frac{\alpha_{m n} \beta_{m n} h_{m n}}{A_{0 m n} h_{l_{m n}}}\right|_{-1+\xi_{m n}^{2}, \alpha_{m n}-1, \beta_{m n}-1} ^{\xi_{m n}^{2}}\right] d h_{m n}
\end{aligned}
$$

By expressing the $\operatorname{erfc}(\cdot)$ as Meijer G function [20, Eq. (8.4.14.2)], the average BER of BPSK-SIM can be expressed in a closed-form utilizing [21, Eq. (21)].

$$
P_{e, M I M O}=\prod_{m=1}^{M} \prod_{n=1}^{N} \frac{2^{\alpha_{m n}+\beta_{m n}-4} \xi_{m n}^{2}}{\sqrt{\pi^{3}} \Gamma\left(\alpha_{m n}\right) \Gamma\left(\beta_{m n}\right)} \times G_{7,4}^{2,6}\left[\left.\frac{4 \gamma^{2} A_{0 m n}^{2} h_{l m n}^{2}}{\sigma^{2} \alpha_{m n}^{2} \beta_{m n}^{2} M^{2} N^{2}}\right|^{\frac{1-\xi_{m n}^{2}}{2}, \frac{2-\xi_{m n}^{2}}{2}, \frac{1-\alpha_{m n}}{2}, \frac{2-\alpha_{m n}}{2}, \frac{1-\beta_{m n}}{2}, \frac{2-\beta_{m n}}{2}, 1} \begin{array}{c}
0,0.5, \frac{-\xi_{m n}^{2}}{2}, \frac{1-\xi_{m n}^{2}}{2}
\end{array}\right]
$$

The above average BER expression is expressed in terms of average SNR as

$$
P_{e, M I M O}=\prod_{m=1}^{M} \prod_{n=1}^{N} \frac{2^{\alpha_{m n}+\beta_{m n}-4} \xi_{m n}^{2}}{\sqrt{\pi^{3}} \Gamma\left(\alpha_{m n}\right) \Gamma\left(\beta_{m n}\right)} \times \mathrm{G}_{7,4}^{2,6}\left[\left.\frac{8 S N R \cdot A_{0 m n}^{2}}{\alpha_{m n}^{2} \beta_{m n}^{2} M^{2} N^{2}}\right|^{\frac{1-\xi_{m n}^{2}}{2}, \frac{2-\xi_{m n}^{2}}{2}, \frac{1-\alpha_{m n}}{2}, \frac{2-\alpha_{m n}}{2}, \frac{1-\beta_{m n}}{2}, \frac{2-\beta_{m n}}{2}, 1} \begin{array}{c}
0,0.5, \frac{-\xi_{m n}^{2}}{2}, \frac{1-\xi_{m n}^{2}}{2}
\end{array}\right]
$$

\section{Numerical results and discussion}

In this section, the explicit statistical results developed from the expressions derived in sections 4 are depicted. For the numerical assessments, the parameters considered for FSO system are, noise standard deviation $\sigma_{n}=10^{-7} \mathrm{~A} / \mathrm{Hz}$, photo detector responsivity $\gamma=0.5 \mathrm{~A} / \mathrm{W}$, beam radius $w_{L} \cong 2.5 \mathrm{~m}$ at $1 \mathrm{~km}$ distance and jitter standard deviation $\sigma_{s} \cong 30 \mathrm{~cm}$. 


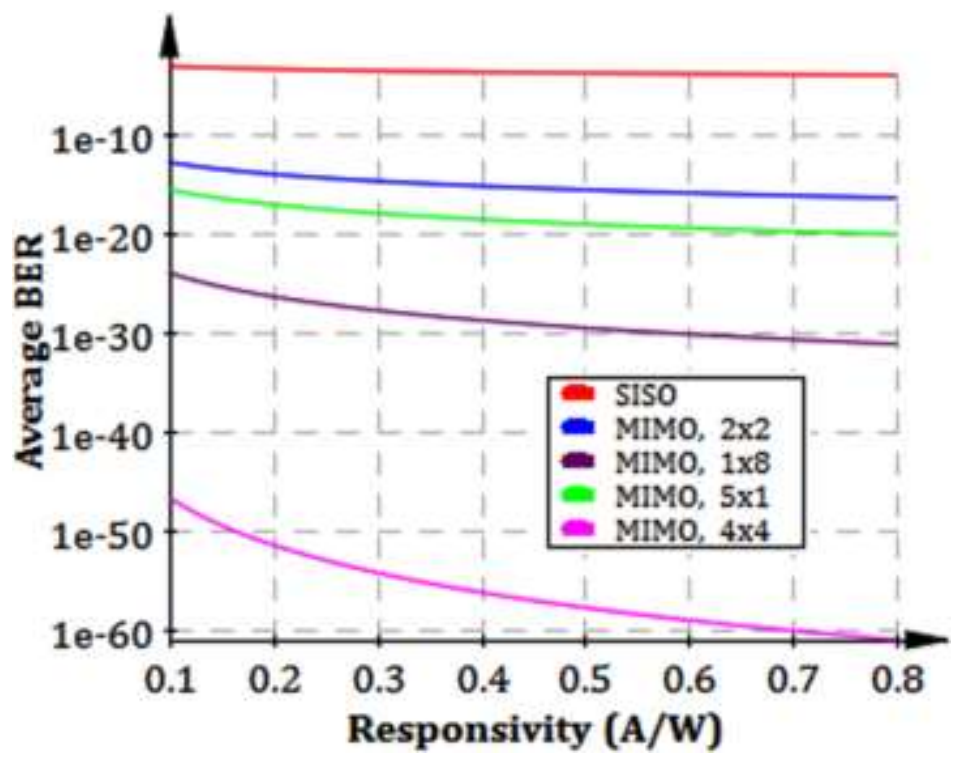

Fig. 1. Average BER against responsivity for SISO and MIMO-FSO systems with $\alpha=4, \beta=1$.

Figs. 1 and 2 are shown to have MIMO system instead of SISO, gives better BER performance against responsitivity. It is also noticed that an improved BER performance along with the responsivity of the photodetector is achieved as the number of transmitting and receiving apertures is enhanced in both moderate $(\alpha=4, \beta=1)$ and strong $(\alpha=1, \beta=1)$ atmospheric turbulences. The best BER for MIMO (4× 4) system is $10^{-58}$ and $10^{-45}$ in moderate and strong atmospheric turbulences respectively with responsivity of $0.5 \mathrm{~A} / \mathrm{W}$.

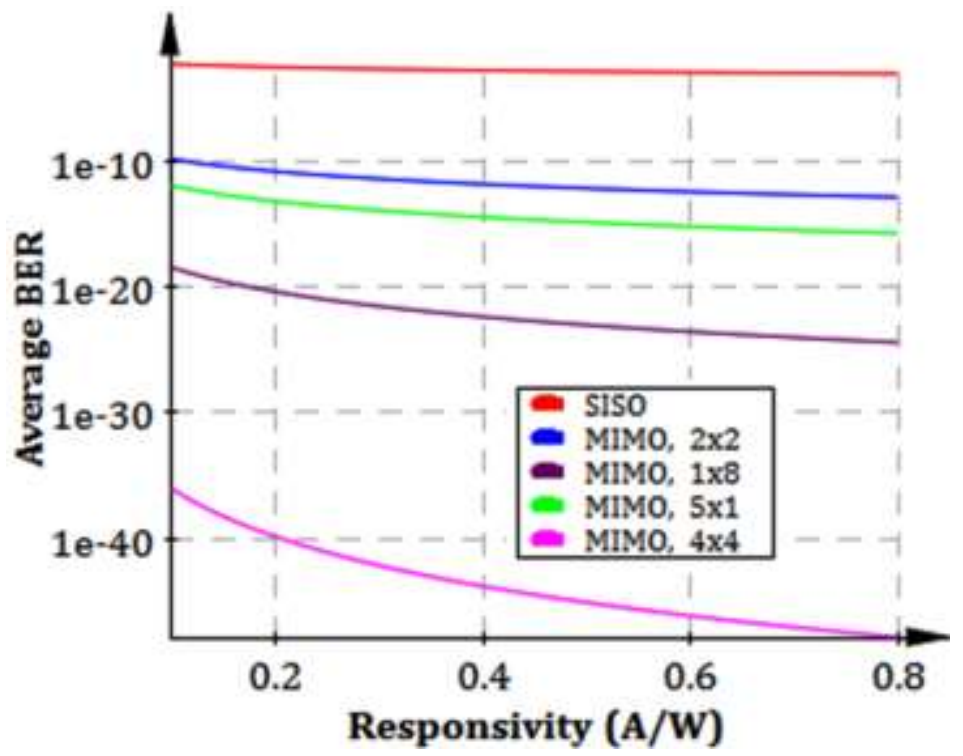

Fig. 2. Average BER against responsivity for SISO and MIMO-FSO systems with $\alpha=1, \beta=1$. 


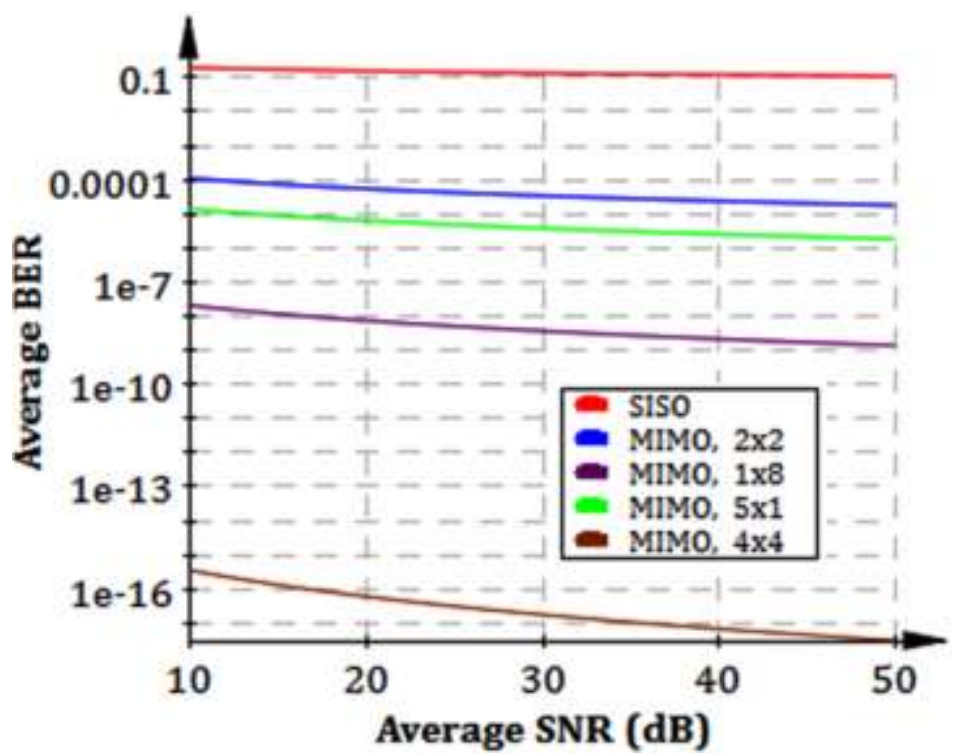

Fig. 3. Average BER against average SNR for SISO and MIMO-FSO systems with $\alpha=4, \beta=1$.

Figs. 3 and 4 illustrate the BER performance with respect to SNR for moderate $(\alpha=4, \beta=1)$ and strong $(\alpha=1, \beta=1)$ atmospheric turbulences respectively. That is, the average BER performance improves drastically with the number of transmitting and receiving apertures. In all the above cases, MIMO system is inferred to have outperformed SISO. Also, the better BER is for MIMO $(4 \times 4)$ system with $10^{-16}$ and $10^{-14}$ in moderate and strong atmospheric turbulences respectively with SNR being $20 \mathrm{~dB}$.

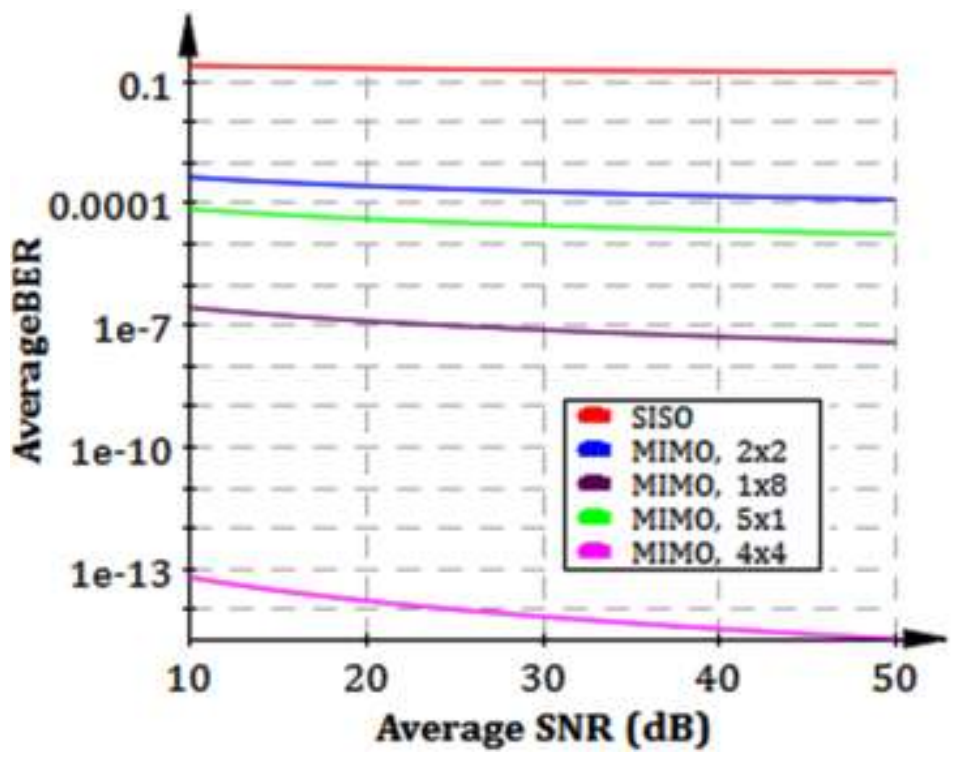

Fig. 4. Average BER against average SNR for SISO and MIMO-FSO systems with $\alpha=1, \beta=1$. 


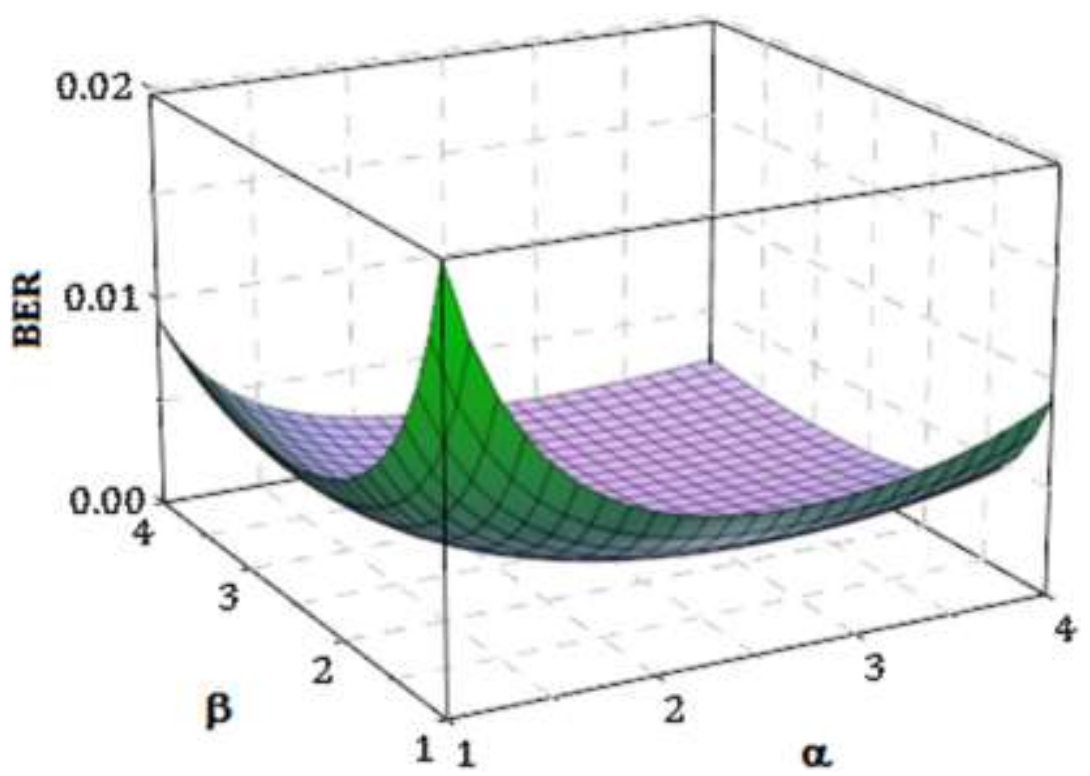

Fig. 5. 3D plot for average BER variation for MIMO-FSO system $(M=1, N=2)$ with respect to $\alpha$ and $\beta$.

The 3D plots are shown in Figs. 5 and 6. A trade-off between the average BER against the effective number of large scale $(\alpha)$ and small scale $(\beta)$ turbulent eddies for SIMO and MIMO systems is achieved. The minimum BER values are $2 \times 10^{-3}$ and $2 \times 10^{-5}$ are obtained for $\mathrm{M}=1, \mathrm{~N}=2$ and $\mathrm{M}=\mathrm{N}=2$ respectively. It is also observed that the better BER performance is achieved for large values of $\alpha=\beta=4$.

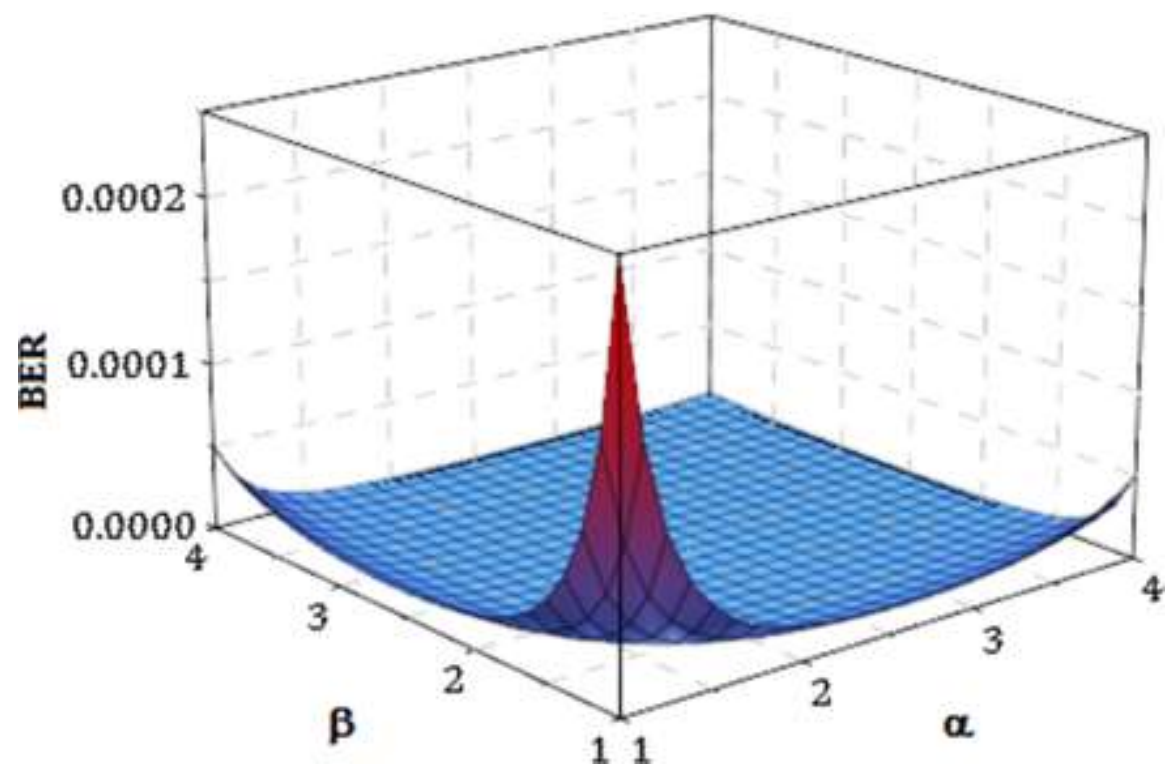

Fig. 6. 3D plot for average BER variation for MIMO-FSO system $(M=2, N=2)$ with respect to $\alpha$ and $\beta$. 


\section{Conclusion}

The exact closed-form expressions for the average BER of BPSK-SIM based SISO and MIMO-FSO systems over a strong atmospheric channel with misalignment are derived. It is observed that MIMO system provides the better BER performance compared to SISO system. It is also seen that better BER performance is achieved by boosting the number of transmitting and receiving apertures. The minimum BER of $10^{-16}$ and $10^{-14}$ are obtained through MIMO technique at $S N R \cong 20 d B$ for $\alpha=4, \beta=1$ and $\alpha=\beta=1$ respectively.

\section{References}

[1] J. M. Alattar and J. M. H. Elmirghani, Optical wireless systems employing adaptive collaborative transmitters in an indoor channel, IEEE Trans. Veh. Technol. 59 (1) (2010) 63-74.

[2] Jitendra Singh, Naresh Kumar, Performance analysis of different modulation format on free space optical communication system, Optik, 124 (20) (2013) 4651-465.

[3] D. Kedar and S. Arnon, Urban optical wireless communications networks: The main challenges and possible solutions, IEEE Commun. Mag. 42 (5) (2003) 2-7.

[4] J. T. Li and M. Uysal, Optical wireless communications: system model, capacity and coding,Vehicular Technol. Conf. 1 (2003) 168-172.

[5] M. Uysal, J. T. Li and M. Yu, Error rate performance analysis of coded free-space optical links over gammagamma atmospheric turbulence channels, IEEE Trans. Wireless Commun. 5 (2006) 1229- 1233.

[6] H. Wu, B. Hamzeh, M. Kavehrad, Availability of airborne hybrid FSO/RF links, Proc. SPIE 5819 (2005) 89-100.

[7] C. Abou-Rjeily, W. Fawaz, Space-time codes for MIMO ultra-wideband communications and MIMO freespace optical communications with PPM, IEEE Journal on Selected Areas in Communications 26 (6) (2008), 938-947. 
[8] E. Bayaki, R. Schober, R. K. Mallik, Performance analysis of MIMO free-space optical systems in gamma-gamma fading, IEEE Transactions on Communications 57(11) (2009), 3415-3424.

[9] T. G. Ulmer, S. R. Henion, F. G. Walther, P. A. Schulz, Differential Phase-Shift Keying in Spatial Diversity Transmitters for Fade Mitigation, IEEE Journal of Selected Topics in Quantum Electronics 16(5) (2010), 1091-1098.

[10] A. A. Farid, S. Hranilovic, Diversity gain and outage probability for MIMO free-space optical links with misalignment, IEEE Transactions on Communications 60 (2) (2012) 479-487.

[11] Z. Chen, S. Yu, T. Wang, G. Wu, H. Guo, W. Gu, Spatial correlation for transmitters in spatial MIMO optical wireless links with Gaussian-beam waves and aperture effects, Optics Communications 287 (15) (2013) 12-18.

[12] M. Niu, J. Cheng, J. F. Holzman, MIMO Architecture for Coherent Optical Wireless Communication: System Design and Performance, Journal of Optical Communications and Networking 5 (5) (2013) 411-420.

[13] K. Prabu, S. Bose, D. Sriram Kumar, BPSK based subcarrier intensity modulated free space optical system in combined strong atmospheric turbulence, Optics Communications 305 (15) (2013) 185-189.

[14] X. M. Zhu and J. M. Kahn, Free-space optical communication through atmospheric turbulence channels, IEEE Trans. Commun., 50(8) (2002) 1293-1300.

[15] A. A. Farid, S. Hranilovic, Outage capacity optimization for free-space optical links with pointing errors, J. Lightwave Technol., 25 (7) (2007) 1702-1710.

[16] H. Samimi, P. Azmi, Subcarrier intensity modulated free-space optical communications in K-distributed turbulence channels, Journal of Optical Communications and Networking 2 (8) (2010) 625-632.

[17] T. A. Tsiftsis, H. G. Sandalidis, G. K. Karagiannidis and M. Uysal, Optical wireless links with spatial diversity over strong atmospheric turbulence channels, IEEE Transactions on Wireless Communications 8 (2009) 951-957. 
[18] W.O. Popoola, Z. Ghassemlooy, J. I. H. Allen, E. Leitgeb, S. Gao, Free-space optical communication employing subcarrier modulation and spatial diversity in atmospheric turbulence channel, IET Optoelectronics 2 (1) (2008) 16-23.

[19] W.O. Popoola, Z. Ghassemlooy, BPSK subcarrier intensity modulated free-space optical communications in atmospheric turbulence, Journal of Lightwave Technology 27 (8) (2009) 967-973.

[20] A. P. Prudnikov, Y.A. Brychkov, O.I. Marichev, Integral and series, More Special Functions, 3, Gordon and Breach Science Publishers, Amsterdam, 1986.

[21] V.S., Adamchikand, O.I., Marichev, The algorithm for calculating integrals of hypergeometric type functions and its realization in REDUCE system, in: Proceedings of the International Conference on Symbolic and Algebraic Computation, Tokyo, Japan, 1990, 212-224. 